\title{
Cancer Biotherapy: The Best is Yet to Come Song Xin*
}

Department of Cancer Biotherapy Center, The Third Affiliated Hospital of Kunming Medical University (Tumor Hospital of Yunnan Province), Kunming, Yunnan, People's Republic of China

In 1984, Dr. Oldham proposed that cancer biotherapy is the fourth treatment modality for patients with cancer and might be useful in conjunction with surgery, chemotherapy and radiotherapy. Meanwhile, the theory of biological response modifier therapy also has been proposed [1]. Cancer biotherapy is a therapeutic strategy that enhances the anticancer potential of the immune system to fight against cancer and attenuates the side effects caused by other treatments [2]. Biotherapy consists of gene therapy, targeted therapy and immunotherapy. Various immunotherapeutic strategies, such as adoptive cellular therapy, anticancer vaccine therapy and cytokines, have been developed. Numerous preclinical and clinical trials have demonstrated that biotherapy in combination with surgery, chemotherapy, radiotherapy or other therapies has greatly increased the cure rate, reduced the side effects and improved the quality of life of cancer patients $[3,4]$.

Interferon and interleukin are the first generation of biological agents for the treatment of cancers, but they have serious adverse effects at a high dosage $[5,6]$. In recent years, great advances have been made to improve the antitumor potential while reducing the adverse effects of biological agents. For instance, ipilimumab [7], a human monoclonal antibody that activates the immune system by targeting CTLA-4, has increased the overall survival rate of melanoma patients by $45.6 \%$ at 12 months, $33.2 \%$ at 18 months, and $23.5 \%$ at 24 months. Sipuleucel-T is the first therapeutic cellular immunotherapy that has prolonged the life of metastatic castration-resistant prostate cancer patients in phase III clinical trials [8], representing a new milestone in cancer immunotherapy. Moreover, a randomized study of cytokineinduced killer (CIK) cell immunotherapy of metastatic renal carcinoma has demonstrated improved progression-free survival and overall survival [9].

Now, more and more novel immunotherapeutic agents have been approved for clinical application through numerous clinical trials. Most importantly, in 2013, cell-based therapeutics was thought to be the "third pillar" of future medicines [10]. With the rapid development of biological treatment technology, biotherapy is regarded as the most promising treatment among the modern therapies for cancer patients due to its long-term antitumor effect as well as relatively low adverse effects. Nevertheless, there are still several factors that limit the speedy development of cancer biotherapies.

First of all, the traditionally classical response criteria of the World Health Organization (WHO) or Response Evaluation Criteria in Solid Tumors (RECIST) might be suboptimal for the efficient evaluation of immunotherapies. As is well known, chemotherapeutic agents often bring about meaningful therapeutic effects including tumor shrinkage in baseline lesions within the initial few weeks. However, owing to the fact that the antitumor effects of immunotherapy are induced by cancer-specific immune responses or modification of native immune processes, the antitumor activity mediated by immunotherapeutic agents may take a longer time and, hence, the clinical response of immunotherapies may extend beyond those of cytotoxic agents and after an initial increase in tumor burden or conventional disease progression. Therefore, a novel criterion to evaluate immunotherapy is required. A phase II clinical trial of ipilimumab in patients with advanced melanoma has been performed, and the new immune-related response criteria have been applied [11]. However, because the response time of treatment varies for different cancers, it is important to confirm the time-point response for the assessment of immunotherapy and the elements to be evaluated. Thus, a great number of clinical trials are required to optimize the criteria for cancer biotherapy.

Moreover, it is difficult to define the optimal dosage and schedule for immunotherapy compared to classic chemotherapy and radiotherapy. Due to the different culture protocols in various laboratories, the maximal number of cultured cells is quite diverse. For example, the clinical data regarding CIK cells show different doses for patients, the total number of CIK cells ranges from $21.9 \times 10^{7}$ to $5.2 \times 10^{10}$, and the cycles of treatment are also distinct [12]. Moreover, there are insufficient clinical trials to demonstrate the relationship between the maximal effective dose and maximal tolerated dose. To accelerate the development of biotherapies, a large number of appropriate clinical trials are urgently needed to test the optimal dose and schedule.

In addition, to date, only one vaccine, sipuleucel-T (Provenge), has been approved for prostate cancer patients. "Despite sipuleucel-T increases the median overall survival by 4.1 months, I don't see it as a significant step forward." said Rosenberg [8]. One of the critical problems is the tolerance to antigens. Tumor cells have the ability to modulate the immune response by downregulating the expression of antigens and the function of regulatory cells [13]. Single antigens may have a limited antitumor effect of a vaccine; thus, a multiantigen is necessary to induce a stronger immune response leading to a significant clinical response. Meanwhile, combinatorial approaches are also required. A phase II clinical trial of ipilimumab plus GMCSF in patients with metastatic melanoma provided positive results, presenting an encouraging precedent for combinatorial therapy [14]. Moreover, as PD1 is another immune checkpoint receptor [15], a phase I clinical trial using a combination of anti-PD1 antibody with ipilimumab in patients with advanced melanoma showed that the objective-response rate for all patients in the concurrent-regimen group was $40 \%$ and clinical activity was observed in $65 \%$ of patients. Impressively, at the maximum doses, $53 \%$ of patients had an objective response with tumor reduction of more than $80 \%$ [16]. Therefore, more phase II or III clinical trials should be performed to further identify the antitumor immune responses of biological agents.

*Corresponding author: Song Xin, Department of Cancer Biotherapy Center, The Third Affiliated Hospital of Kunming Medical University (Tumor Hospital of Yunnan Province), Kunming, Yunnan 650031, People's Republic of China, Tel: 868-718100-739; Fax: 868-718-100-739; E-mail: songxin68@126.com

Received September 23, 2013; Accepted September 25, 2013; Published September 27, 2013

Citation: Xin S (2013) Cancer Biotherapy: The Best is Yet to Come. J Cancer Sci Ther 5: e128. doi:10.4172/1948-5956.1000e128

Copyright: ( 2013 Xin S. This is an open-access article distributed under the terms of the Creative Commons Attribution License, which permits unrestricted use, distribution, and reproduction in any medium, provided the original author and source are credited. 
Last but not the least, "translational medicine" should be highlighted to promote the transition from fundamental studies to clinical applications. More and more biological agents have gained a favorable anticancer effect in laboratories; however, insufficient knowledge of the pharmacology and clinical applications has hampered the further development of these agents. Furthermore, biological agents are relatively expensive at the initial period of study and are always given to a limited number of patients, which is another significant reason that slows down the development of biotherapies in clinical applications.

In summary, great advances have been made in cancer biotherapy in the past years. The promise of this exciting and evolving field is exceedingly anticipated and will impart a new vigor to comprehensive cancer treatment. However, in order to accelerate its fast development, it is time to formulate new response criteria for the evaluation of biotherapies. In the long term, a large number of appropriate clinical trials must be carried out to provide evidence-based data.

\section{References}

1. Oldham RK (1984) Biologicals and biological response modifiers: fourth modality of cancer treatment. Cancer Treat Rep 68: 221-232.

2. Kuroki M, Miyamoto S, Morisaki T, Yotsumoto F, Shirasu N, et al. (2012) Biological response modifiers used in cancer biotherapy. Anticancer Res 32 2229-2233.

3. Huang ZM, Li W, Li S, Gao F, Zhou QM, et al. (2013) Cytokine-induced killer cells in combination with transcatheter arterial chemoembolization and radiofrequency ablation for hepatocellular carcinoma patients. J Immunother 36: $287-293$.

4. Iliopoulou EG, Kountourakis P, Karamouzis MV, Doufexis D, Ardavanis A, et al. (2010) A phase I trial of adoptive transfer of allogeneic natural kille cells in patients with advanced non-small cell lung cancer. Cancer Immunol Immunother 59: 1781-1789.

5. Bennett CL, Westbrook CA, Gruber B, Golomb HM (1986) Hairy cell leukemia and mucormycosis. Treatment with alpha-2 interferon. Am J Med 81: 1065 1067.

6. Sleijfer DT, Janssen RA, Buter J, de Vries EG, Willemse PH, et al. (1992) Phase II study of subcutaneous interleukin-2 in unselected patients with advanced renal cell cancer on an outpatient basis. J Clin Oncol 10: 1119-1123.

7. Hodi FS, O'Day SJ, McDermott DF, Weber RW, Sosman JA, et al. (2010) Improved survival with ipilimumab in patients with metastatic melanoma. N Engl J Med 363: 711-723.

8. Kantoff PW, Higano CS, Shore ND, Berger ER, Small EJ, et al. (2010) Sipuleucel-T immunotherapy for castration-resistant prostate cancer. N Engl J Med 363: 411-422.

9. Liu L, Zhang W, Qi X, Li H, Yu J, et al. (2012) Randomized study of autologous cytokine-induced killer cell immunotherapy in metastatic renal carcinoma. Clin Cancer Res 18: 1751-1759.

10. Fischbach MA, Bluestone JA, Lim WA (2013) Cell-based therapeutics: the next pillar of medicine. Sci Transl Med 5: 179ps7.

11. Wolchok JD, Hoos A, O'Day S, Weber JS, Hamid O, et al. (2009) Guidelines for the evaluation of immune therapy activity in solid tumors: immune-related response criteria. Clin Cancer Res 15: 7412-7420.

12. Hontscha C, Borck Y, Zhou H, Messmer D, Schmidt-Wolf IG (2011) Clinical trials on CIK cells: first report of the international registry on CIK cells (IRCC). $J$ Cancer Res Clin Oncol 137: 305-310.

13. Azvolinsky A (2013) Cancer vaccines: always a bridesmaid, never a bride? J Natl Cancer Inst 105: 248-249.

14. [No authors listed] (2013) GM-CSF/Ipilimumab combination extends melanoma survival. Cancer Discov 3: OF6.

15. Lipson EJ (2013) Re-orienting the immune system: Durable tumo regression and successful re-induction therapy using anti-PD1 antibodies. Oncoimmunology 2: e23661.

16. Wolchok JD, Kluger H, Callahan MK, Postow MA, Rizvi NA, et al. (2013) Nivolumab plus ipilimumab in advanced melanoma. N Engl J Med 369: 122133 